\title{
Repeatability and Accuracy of Ankle Centre Location Estimates Using a Biaxial Joint Model
}

\author{
Cameron A. Shute \& Antony J. Hodgson \\ Department of Mechanical Engineering \\ University of British Columbia, Vancouver, BC, Canada \\ ahodgson@mech.ubc.ca
}

\begin{abstract}
In conventional total knee replacement (TKR) surgery, a significant fraction of implants have varus/valgus alignment errors large enough to reduce the lifespan of the implant, so we are developing a more accurate computerassisted procedure aimed at reducing the standard deviation (SD) of the implant procedure. In this study we introduce a new method of locating the ankle joint centre (AJC) using a biaxial model (BM), and determine the accuracy and repeatability of this protocol compared to a digitization method and a spherefitting method used in a current computer-assisted procedure. Repeated in vivo measurements performed by a single operator were obtained from five normal subjects (450 measurements) using the three methods of AJC location. Based on these experiments we estimate the varus/valgus SD of defining the tibial mechanical axis in the frontal plane for the tested population to be $0.28^{\circ}$ for the spherical model, $0.17^{\circ}$ for the biaxial model, and $0.11^{\circ}$ for the conventional digitizing point probe. The mean joint centre locations found by the motionbased models are significantly medial and anterior to the point probe centre.
\end{abstract}

\section{Introduction}

We are developing computer-assisted total knee replacement tooling that eliminates intramedullary rods and improves alignment accuracy without introducing additional imaging requirements (such as preoperative computed tomography scans) or invasive procedures (such as bone pins remote to the operating site). Currently there is no universally accepted method of defining the AJC, but there are two main approaches: anatomical determination (digitization) and biomechanical determination (motionbased). This work investigates the feasibility of using a biaxial model to define a biomechanically meaningful AJC by comparing the centre to those centres obtained with a spherical model and by direct digitization.

\section{Methods}

Digitization measurements were obtained using a $135 \mathrm{~mm}$ point probe, and calcaneal motion measurements were recorded with a optoelectronic three-emitter reference frame mounted to a calcaneal tracker (Flashpoint 5000 localizer). All measurements were relative to a three-emitter triangular local reference frame (120 $\mathrm{mm}$ on a side) mounted rigidly to a tibial tracker. 
Study design: A simulated computer-assisted TKR setting was constructed, and a single operator performed 30 repeated trials on five live subjects (male, mean age 24, mean weight $80 \mathrm{~kg}$ ), digitizing what was considered to be the extremes of both medial and lateral malleoli. Subjects then performed 30 trials of the following movements: dorsi/plantarflexion with the ankle in the neutral, inverted and everted positions, in/eversion with the ankle in the dorsiflexed, neutral and plantarflexed positions, and finally circumduction. The measured data was then used to fit a 12 parameter biaxial joint model developed previously [Bogert 1994]. The same motion data was also used to determine the AJC using a sphere-fitting method.

\section{Results and Discussion}

Table 1 summarizes the results of the experiment; the digitizing point probe method had significantly higher precision than either motion-based method in the frontal plane $(\mathrm{P}=0.002$ Biaxial, $\mathrm{P}=0.0003$ Spherical). The biaxial method was significantly less variable than the spherical method $\left(0.17^{\circ} \mathrm{SD}\right.$ vs $\left.0.28^{\circ} \mathrm{SD}, \mathrm{P}=0.004\right)$.

Table 1. Repeatability of joint centre location in the frontal plane

\begin{tabular}{|c|c|c|c|c|}
\hline $\begin{array}{c}\text { AJC Location } \\
\text { Method }\end{array}$ & $\begin{array}{c}\text { ML Direction } \\
\text { SD }(\mathrm{mm})\end{array}$ & $\begin{array}{c}\text { Varus/Valgus } \\
\mathrm{SD}\left({ }^{\circ}\right)\end{array}$ & $\begin{array}{c}\text { Bias relative to point } \\
\text { probe centre }(\mathrm{mm})\end{array}$ & $\begin{array}{c}\text { Range } \\
(\mathrm{mm})\end{array}$ \\
\hline Point Probe & 0.69 & 0.11 & N/A & 2.75 \\
Biaxial Method & 1.14 & 0.17 & 7.24 (medial) & 5.00 \\
Sphere Method & 1.84 & 0.28 & 10.80 (medial) & 7.23 \\
\hline
\end{tabular}

Varus/valgus alignment appears to have a strong effect on implant lifespan, so the repeatability of ankle centre identification is a key design parameter in computerassisted TKR procedures. This is the first report on the precision of locating an AJC using a biaxial joint model.

Although the repeatability of locating the AJC with the point probe is significantly better than the motion-based methods, the anatomic centre so defined is only indirectly related to the loads carried by the implant. The kinematic centres defined by either the biaxial or spherical models are more relevant for load calculations.

Since only five subjects were tested in this preliminary study, we do not yet have enough data to say that the kinematically-determined centres are not colocated with the anatomical centres. However, the AJCs found using the motion-based methods were biased medially $(7-11 \mathrm{~mm})$ and slightly anteriorly $(1-3 \mathrm{~mm})$. A medial shift in the functional joint centre would imply that the knee is in greater varus than previously suspected and implants are known to tolerate varus alignments poorly.

\section{References}

1. van den Bogert AJ, Smith GD, Nigg BM: In Vivo Determination of the Anatomical Axes of the Ankle Joint Complex: an Optimization Approach. Journal of Biomechanics 27(12), 1994. pp 1477-1488. 